\title{
A Forefoot Strike Requires the Highest Forces Applied to the Foot Among Foot Strike Patterns
}

\section{(c) () $\$)_{\text {Version } 1}$}

\section{Authors}

Satoru Hashizume ${ }^{1}$, Toshio Yanagiya²

Affiliations

1 National Institute of Advanced Industrial Science, Human Informatics Research Institute, Tokyo, Japan

2 Faculty of Health and Sports Science, Juntendo University, Chiba, Japan

Key word

running, moment arm, Achilles tendon force, joint reaction force, musculoskeletal injury

accepted after revision November. 14. 2016

\section{Bibliography}

DOI http://dx.doi.org/10.1055/s-0042-122017

Published online: 2017 | Sports Medicine International Open 2017;

1: E37-E42

(c) Georg Thieme Verlag KG Stuttgart · New York

ISSN 2367-1890

Correspondence

Dr. Satoru Hashizume, PhD

National Institute of Advanced Industrial Science

Human Informatics Research Institute
Waterfront 3F, 2-3-26 Aomi, Koto-ku

135-0064 Tokyo

Japan

Tel.: +81/3/35998 503, Fax: +81/3/55005 233

satoru.hashizume@aist.go.jp

\begin{abstract}
Ground reaction force is often used to predict the potential risk of injuries but may not coincide with the forces applied to commonly injured regions of the foot. This study examined the forces applied to the foot, and the associated moment arms made by three foot strike patterns. 10 male runners ran barefoot along a runway at $3.3 \mathrm{~m} / \mathrm{s}$ using forefoot, midfoot, and rearfoot strikes. The Achilles tendon and ground reaction force moment arms represented the shortest distance between the ankle joint axis and the line of action of each force. The Achilles tendon and joint reaction forces were calculated by solving equations of foot motion. The Achilles tendon and joint reaction forces were greatest for the forefoot strike ( 2194 and $3137 \mathrm{~N}$ ), followed by the midfoot strike (1 929 and $2853 \mathrm{~N}$ ), and the rearfoot strike (1526 and $2394 \mathrm{~N}$ ). The ground reaction force moment arm was greater for the forefoot strike than for the other foot strikes, and was greater for the midfoot strike than for the rearfoot strike. Meanwhile, there were no differences in the Achilles tendon moment arm among all foot strikes. These differences were attributed mainly to differences in the ground reaction force moment arm among the three foot strike patterns.
\end{abstract}

\section{Introduction}

Distance running is known to reduce the risks of some health problems in runners [7, 27, 31, 41]; however, distance running also has negative effects that result in musculoskeletal overuse injuries in the lower extremities. The incidence of running-related musculoskeletal overuse injuries in previous studies ranged from 20 to $79 \%$ [39]. The lower leg, foot, and ankle, especially the Achilles tendon and tibia, are commonly affected in running-related injuries $[19,24,26,38]$. On the basis of material fatigue, large forces applied to injured regions will decrease the number of cycles to failure [3]. Therefore, a decrease in the force applied to the lower leg, foot, and ankle during running may be able to reduce the potential risk of running-related injuries at the Achilles tendon and the tibia for a given running distance and frequency.

Foot strike pattern is generally classified into 3 types according to the ground reaction force (GRF) application point, relative to foot length, at the instance of foot contact [5]. Foot strike pattern has been thought to influence the potential risk of running-related injuries $[8,21,23,36]$. Previous studies reported that $69-75 \%$ runners use the rearfoot strike (RFS) and 25-31\% runners use the midfoot strike (MFS) or rearfoot strike (RFS) $[8,15]$. It was reported that the vertical component of GRF during the early stance phase, and the loading rate of that force, were smaller for FFS and MFS than for $\operatorname{RFS}[5,22,23,28]$. In these studies, it was suggested that FFS might reduce the potential risk of injury as compared with RFS. However, the GRF does not necessarily coincide with the forces applied to the Achilles tendon and the tibia, and the magnitude of forces applied to the Achilles tendon and the tibia may be much greater than the GRF $[9,20,32,34]$. Although previous studies suggested that measurements of GRF may not be sufficient to evaluate the potential risk of running-related injuries, it is difficult to directly measure the forces applied to the Achilles tendon and the tibia.

An inverse dynamics approach might resolve this problem. The forces applied to the Achilles tendon and the tibia can be calculated as the Achilles tendon force and joint reaction force, respectively. At the instance of foot contact, the application point of the GRF is positioned at the ball of the foot for FFS, the middle of the foot for MFS, and the heel for RFS, respectively [5]. When the orientation of the GRF line of action is similar among the three foot strike patterns, the GRF moment arm, defined as the shortest distance between the ankle joint axis and the GRF line of action, is greater for FFS than the other two foot strike patterns. A difference in the GRF moment arm induces a difference in the moment of the GRF, 
and differences in this moment may also induce differences in the Achilles and joint reaction forces. Furthermore, the Achilles tendon moment arm also affects the relationships among the GRF, the Achilles tendon force and the joint reaction force. However, there are only limited studies examining the influence of foot strike patterns on these kinetic parameters by using an inverse dynamics approach $[21,29,40,43]$, and the influence of three foot strike patterns on the Achilles tendon and joint reaction forces and the associated moment arm remains unclear.

Clarifying the influences of three foot strike pattern on the forces applied to foot may help to reduce the potential risks of injuries occurring to the Achilles tendon and the tibia. We therefore hypothesized that the Achilles tendon and joint reaction forces are greater for FFS than for MFS, that forces for MFS would be greater than for RFS, and that these differences may originate from differences in the GRF moment arm associated with foot strike pattern. Thus, the purpose of this study was to examine the influence of three foot strike patterns on the forces applied to the foot and the associated moment arm of the forces.

\section{Materials \& Methods}

\section{Subjects}

Ten young adult male recreational runners without musculoskeletal injuries in the lower extremities participated in this study (age: $21.0 \pm 1.8$ years old, height: $1.73 \pm 0.08 \mathrm{~m}$, body mass: $62.7 \pm 5.0 \mathrm{~kg}$ ). Running experience, weekly running frequency, and weekly running distance were $6.5 \pm 2.6$ years, $5.4 \pm 1.1$ days, and $83.8 \pm 42.7 \mathrm{~km}$, respectively. Written informed consent was obtained from each subject before the experiment. The experimental protocol of this study was approved by the Ethics Committee on Human Research of Juntendo University. This study was conducted in accordance with the Declaration of Helsinki and meets the ethical standards of the International Journal of Sports Medicine [14].

\section{Data collection}

Eight retro-reflective markers were attached to the following anatomical landmarks of the right lower extremity: 1) medial epicondyle, 2) lateral epicondyle, 3) medial malleolus, 4) lateral malleolus, 5) calcaneal tuberosity, 6) distal muscle-tendon junction of the soleus muscle, 7 ) head of the second metatarsal bone and 8) tip of the second finger. Before attaching the markers, the position of the distal muscle-tendon junction of the soleus muscle was identified by using an ultrasound apparatus (SSD-4000, Aloka, Japan).

The experiments were performed in a room with a straight $15-m$ runway for subjects to run. Subjects were asked to perform barefoot running with FFS, MFS, and RFS at a speed of $3.3 \mathrm{~m} \cdot \mathrm{s}^{-1}$. This speed corresponds to the typical running speed for recreational runner [30]. Before the experimental trials, subjects were given self-selected warm-up and practice running trials to adapt each foot strike pattern to their natural running form. 5 successful trials were recorded during the stance phase of running for each foot strike pattern. Those trials in which 1) running speed during, before, and after the force platform at a distance of $2 \mathrm{~m}$ was not within $5 \%$ of the target running speed; 2 ) the subject's right foot did not contact the force platform; and 3) the subject identified that the running form used in the trial was not natural were rejected. The order of the three foot strike patterns was set randomly for each subject. For each trial, the three-dimensional coordinates of markers were recorded at $250 \mathrm{~Hz}$ using an optical motion capture system with 8 cameras (VICON MX system, Oxford Metrics Ltd, UK). The GRF was recorded simultaneously at $1000 \mathrm{~Hz}$ using a force platform system (9287C/CA, Kistler Instruments Ltd, Switzerland). Before the beginning of each experiment, these systems were calibrated using VICON Nexus software (Oxford Metrics Ltd, UK) in order to define a common global coordinate system. The running speeds were $3.29 \pm 0.02 \mathrm{~m} \cdot \mathrm{s}^{-1}, 3.32 \pm 0.04 \mathrm{~m} \cdot \mathrm{s}^{-1}$ and $3.29 \pm 0.04 \mathrm{~m} \cdot \mathrm{s}^{-1}$ for FFS, MFS, and RFS, respectively. In this study, subjects were instructed to use three different foot strike patterns for running trials. We therefore confirmed that subjects could use each foot strike pattern appropriately for each trial. Previously, FFS, MFS, and RFS were defined as conditions where the GRF application points were in the front one-third, middle one-third, and rear one-third of foot length at the instance of foot contact, respectively [4]. Therefore, the ratio of the foot length and the heel-to-GRF application point length at the instance of foot contact defined those foot strike patterns. The ratios for FFS, MFS, and RFS were $0.87 \pm 0.12,0.39 \pm 0.12$ and $0.17 \pm 0.04$, respectively, confirming that the subjects were able to control foot strike patterns according to instructions.

\section{Data reduction}

The collected data were filtered using a fourth-order Butterworth low-pass filter. The cut-off frequencies for each data set were calculated using the method proposed by Yu and Hay [44]. The ankle joint axis was defined as the line passing through the medial and lateral malleoli. The line of action of the Achilles tendon force was defined as the line passing through the calcaneal tuberosity and the distal muscle-tendon junction of the soleus muscle. The Achilles tendon force line of action was projected to the orthogonal plane of the ankle joint axis, and then the shortest distance between that projected line and the ankle joint axis was calculated as the Achilles tendon moment arm [6, 16-18, 35]. Inertia parameters of the foot were calculated by using the method proposed by Ae et al. [1]. The GRF and foot weight moment arms were calculated as similar to the Achilles tendon moment arm.

The Achilles tendon and joint reaction forces $\left(\vec{F}_{A T}\right.$ and $\vec{F}_{\text {joint }}$, respectively) were calculated by resolving the motion of foot equations. The right foot was modeled as a rigid body, based on a previous study [33], and described in the free-body diagram in > Fig. 1. The linear and angular motion of foot equations were written as follows:

$$
\begin{aligned}
& \sum \vec{M}_{a x i s}=\vec{M}_{G R F}+\vec{M}_{A T}+\vec{M}_{W}=I_{\text {foot }} \ddot{\vec{\theta}}_{\text {foot }} \\
& \sum \vec{F}_{\text {foot }}=\vec{F}_{G R F}+\vec{F}_{A T}+\vec{F}_{\text {joint }}+\vec{W}_{\text {foot }}=m_{\text {foot }} \ddot{\vec{d}}_{\text {foot }}
\end{aligned}
$$

where $\vec{M}_{G R F}, \vec{M}_{A T}$ and $\vec{M}_{W}$ represent the moments of the GRF, Achilles tendon force and foot weight, respectively; $\ddot{\vec{\theta}}_{\text {foot }}$ represents the angular acceleration of the ankle joint; $\vec{F}_{G R F}$ and $\vec{W}_{\text {foot }}$ represent the GRF and the foot weight, respectively; and $\ddot{\vec{d}}_{\text {foot }}$ represents the linear displacement of the center of foot mass. Positive and negative values of moment around the ankle joint axis represent- 


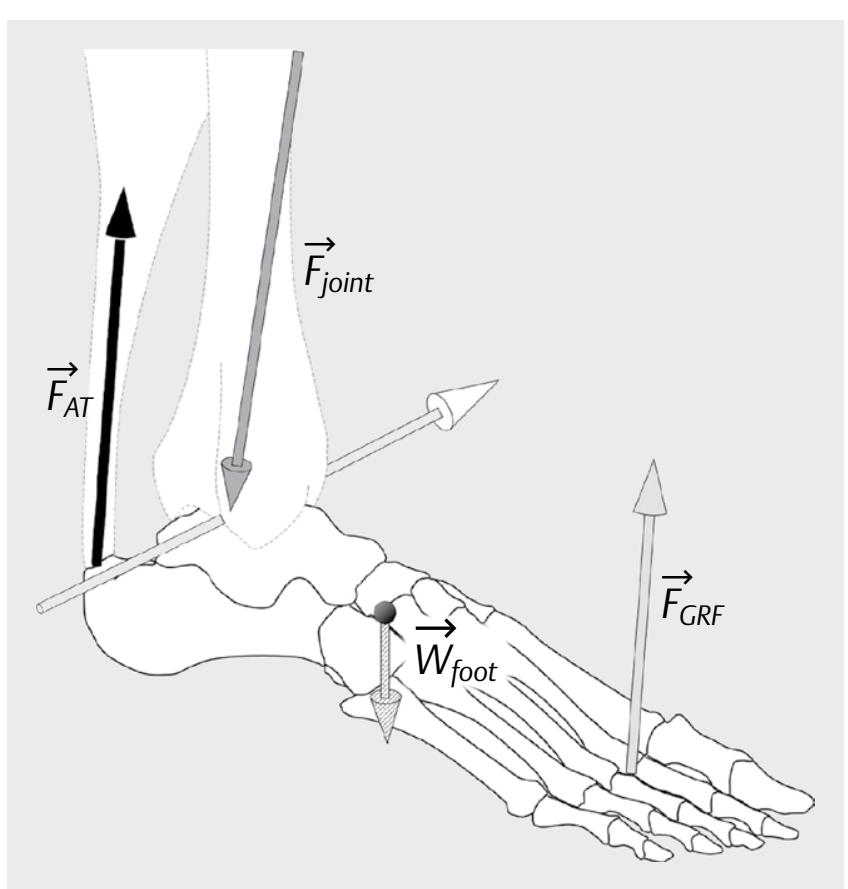

- Fig. 1 Free-body diagram of the foot. $\vec{F}_{G R F}$ ground reaction force; $\vec{F}_{A T}$, Achilles tendon force; $\vec{F}_{\text {joint }}$, joint reaction force; and $\vec{W}_{\text {foot }}$, foot weight.

ed plantar-flexion and dorsiflexion moments, respectively. The calculated time-series data during the contact phase of running were averaged for each parameter.

\section{Statistics}

The normality of each parameter was confirmed by the Kolmogorov-Smirnov test $(p>0.05)$. A one-way analysis of variance (ANOVA) with a Bonferroni post hoc test was used to test the difference in each parameter among three foot strike patterns. Effect size was calculated as partial $\eta^{2}$. The level of significance was set at 0.05 . These statistical analyses were executed using statistical software (IBM SPSS Statistics Version 22.0, SPSS Inc, US).

\section{Results}

The means and standard deviations of each parameter were listed in $>$ Table 1 . The typical time-series data of each force were represented in $>$ Fig. 2. Each force was represented as the absolute value. The GRF was significantly greater for FFS and MFS than for RFS, whereas there was no difference between FFS and MFS. The Achilles tendon and joint reaction forces were significantly greater for FFS than for the other two foot strikes, and the forces for MFS were significantly greater than for RFS.

The moment of the GRF was significantly smaller for FFS than the other two foot strikes, and was smaller for MFS compared to RFS. In contrast, the moment of the Achilles tendon force was significantly greater for FFS than the other two foot strikes, and was greater for MFS compared to RFS. The moment of foot weight was not different among the foot strike patterns.

The GRF moment arm was significantly greater for FFS than for the other two foot strike patterns, and for MFS for RFS. Meanwhile, the Achilles tendon moment arm was not different among the foot strike patterns. The foot weight moment arm was significantly smaller for FFS than the other two foot strike patterns, and there was no difference in this value between MFS and RFS.

\section{Discussion}

We examined the influence of three different foot strike patterns on the forces applied to the foot and the associated moment arms during barefoot running. The major original findings of this study were that the Achilles tendon and joint reaction forces were greatest for FFS, and were greater in MFS than in RFS. We also demonstrated that the GRF moment arm was greatest for FFS, and was greater for MFS than for RFS. These results support our hypothesis.

Our results revealed that the Achilles tendon and joint reaction forces increased from RFS to MFS to FFS, and that these trends did not correlate with changes in the GRF. Previous studies confirmed the influence of foot strike pattern on the GRF and suggested that FFS may be able to reduce the potential risk of running-related injuries occurring at lower extremities [22,23]. Present results, however, suggest that using GRF to evaluate potential risk factors of running-related injuries occurring at the Achilles tendon and the tibia may not be sufficient. Musculoskeletal overuse injuries generally occur because of cumulative, repetitive microtraumas to an injured body part. The large Achilles tendon force, therefore, can put the Achilles tendon at the potential risk of an overuse injury such as tendinopathy, and the large joint reaction force can put the tibia at the potential risk of stress fractures [10]. This data suggests that the potential risks of such running-related injuries are greatest for FFS, followed by MFS, then RFS.

The joint reaction force was $10 \%$ greater for FFS than MFS, and it was $19 \%$ greater for MFS than for RFS. Mechanically, the differences in joint reaction force among the three foot strike patterns can be explained by the other forces applied to foot. Foot weight is not affected by foot strike patterns. Meanwhile, the GRF was $3 \%$ greater in FFS than in MFS, and the GRF was also $4 \%$ greater for MFS than for RFS, which is similar to previous studies [5, 21, 29] that reported that the vertical component of GRF was slightly greater for FFS and MFS than RFS. It is not surprising that GRF results were similar between the present and previous studies, because the major component of GRF is not the anteroposterior or mediolateral components, but the vertical component. Meanwhile, the Achilles tendon force was $14 \%$ greater in FFS than in MFS, and $26 \%$ greater in MFS than in RFS. Previous studies $[2,41]$ simulated the Achilles tendon force by using a forward dynamics approach, and they reported that this force was greater for non-RFS than for RFS. Our results using an inverse dynamics approach support the published forward dynamics approaches, and provide additional information about differences in Achilles tendon force between FFS and MFS. The difference in the joint reaction force among the three foot strike patterns, therefore, was mainly attributable to the difference in the Achilles tendon force.

The difference in Achilles tendon force among the three foot strike patterns can also be explained by differences in other mechanical parameters. The Achilles tendon force was calculated by dividing the moment of the Achilles tendon force by the Achilles 
- Table 1 The means and standard deviations of calculated parameters for each foot strike pattern.

\begin{tabular}{|c|c|c|c|c|c|}
\hline & FFS & MFS & RFS & $P$ value & partial $\eta^{2}$ \\
\hline GRF (N) & $1028 \pm 117^{\dagger}$ & $1001 \pm 107^{\dagger}$ & $962 \pm 93^{* \#}$ & $<0.01$ & 0.45 \\
\hline Achilles tendon force $(\mathrm{N})$ & $2194 \pm 208^{\# \dagger}$ & $1929 \pm 238^{*} \dagger$ & $1526 \pm 208^{* \#}$ & $<0.01$ & 0.79 \\
\hline Joint reaction force $(\mathrm{N})$ & $3137 \pm 267 \# \dagger$ & $2853 \pm 316^{*} \dagger$ & $2394 \pm 219^{* \#}$ & $<0.01$ & 0.76 \\
\hline Moment of the GRF $(\mathrm{N} \cdot \mathrm{m})$ & $-109.6 \pm 13.4^{\# \dagger}$ & $-94.2 \pm 12.3^{*} \dagger$ & $-77.4 \pm 6.3^{* \#}$ & $<0.01$ & 0.78 \\
\hline Moment of the Achilles tendon force $(\mathrm{N} \cdot \mathrm{m})$ & $107.0 \pm 14.2^{\# \dagger}$ & $91.1 \pm 13.4^{* \dagger}$ & $76.6 \pm 6.8^{* \#}$ & $<0.01$ & 0.75 \\
\hline Moment of the foot weight $(\mathrm{N} \cdot \mathrm{m})$ & $0.2 \pm 0.0$ & $0.2 \pm 0.0$ & $0.2 \pm 0.0$ & 0.23 & 0.15 \\
\hline GRF moment arm (mm) & $110 \pm 6 \# \dagger$ & $96 \pm 7^{* \dagger}$ & $77 \pm 9^{*} \#$ & $<0.01$ & 0.82 \\
\hline Achilles tendon moment arm $(\mathrm{mm})$ & $52 \pm 4$ & $51 \pm 4$ & $51 \pm 4$ & 0.23 & 0.3 \\
\hline Foot weight moment arm $(\mathrm{mm})$ & $23 \pm 2 \# \dagger$ & $26 \pm 2^{*}$ & $26 \pm 2^{*}$ & $<0.01$ & 0.55 \\
\hline
\end{tabular}

tendon moment arm. The moment of the Achilles tendon force was $18 \%$ greater for FFS than MFS and $19 \%$ greater for MFS than RFS. Meanwhile, the Achilles tendon moment arm was not different among the three foot strike patterns. The difference in the Achilles tendon force among the three foot strike patterns, therefore, cannot be explained by a difference in the Achilles tendon moment arm but by the difference in the moment of the Achilles tendon force.

The moment of the Achilles tendon force may be influenced by the moment of other forces. Our result showed that the moment of the GRF was $16 \%$ smaller for FFS than MFS and $19 \%$ smaller for MFS than RFS. There is no study, to our knowledge, that has examined the influence of three foot strike patterns on the moment of the GRF, but Kulmara et al. [21] confirmed the difference in the moment of the GRF between FFS and RFS. They compared FFS and RFS in two groups of female runners and reported that the dorsiflexion moment of the GRF in the sagittal plane was greater for FFS than RFS. Although subject characteristics were different between present and previous studies, the result associated with the moment of the GRF accorded well. Meanwhile, the moment of the foot weight was not different among the three foot strike patterns. The difference in moment of the Achilles tendon force among the three foot strike patterns, therefore, is mainly attributable to the difference in the moment of the GRF.

The difference in moment of the GRF among the three foot strike patterns was thought to result from differences in the GRF and the GRF moment arm. As mentioned earlier, the differences in GRF among the three foot strike patterns were not large, suggesting that GRF may have little effect on the moment of the GRF. Meanwhile, the GRF moment arm was $15 \%$ greater in FFS than MFS, and $25 \%$ greater for MFS than RFS. These differences in GRF moment arm were induced by differences in the application point of the GRF. As mentioned earlier, the ratio of the GRF application point relative to foot length at the instance of foot contact were $0.87,0.39$ and 0.17 for FFS, MFS and RFS, respectively. Therefore, the difference in moment of the GRF among the three foot strike patterns was mainly attributable to the difference in the GRF moment arm due to the difference in the application point of the GRF.

The major limitations of this study associated with the methods should be mentioned for interpretation of the present findings. One limitation was that we did not take into account the effect of the forces exerted by the dorsiflexor muscles. Shih et al [36] reported that the tibialis anterior muscle activation level was $8.5 \%$ greater for RFS than for FFS during the contact phase of barefoot running. Previous studies $[12,25]$ have reported that the maximal voluntary dorsiflexion moment is about $15-45 \mathrm{~N} \cdot \mathrm{m}$. Therefore, an $8.5 \%$ difference in the tibialis anterior muscle activation level may induce a $1-4 \mathrm{~N} \cdot \mathrm{m}$ difference in the dorsiflexion moment. Because the differences in the moment of the GRF among the three foot strike patterns observed in this study were larger than $4 \mathrm{~N} \cdot \mathrm{m}$ ( $\triangleright$ Table 1), this limitation may have little effect on the main findings of this study. Furthermore, our study determined the Achilles tendon moment arm using retro-reflective markers. The three-dimensional method using magnetic resonance imaging (MRI) has been thought to determine the Achilles tendon moment arm accurately [16, 17], because MRI can identify the actual positions and orientations of bones and the Achilles tendon. Previous studies [6, 18, 35] determined the Achilles tendon moment arm during muscle contraction condition by using MRI. Nonetheless, the Achilles tendon moment arm determined in this study $(51-52 \mathrm{~mm})$ was within the values reported in previous studies $(47-55 \mathrm{~mm}$ ), suggesting that this limitation has little effect on the main findings of this study. Furthermore, there are certain considerations that should be acknowledged when interpreting the results of the current study. Young male runners participated in this study, and they were asked to perform barefoot running along a level runway at $3.3 \mathrm{~m} \cdot \mathrm{s}^{-1}$. Gender [11], age [9, 37], footwear [42], ground surface [4], gradient [13], and running speed [28] may have potential to affect present results. Some studies did not determine the forces applied to injured regions but ground reaction force and/or joint moment. Further experiments are required to test the influences of these factors on the relationships between foot strike patterns and the forces applied to the injured region. These studies may advance and expand the main finding of present study.

In summary, we first examined the influence of three foot strike patterns on the forces applied to the foot and the associated moment arms. Our results showed that the Achilles tendon and joint reaction forces were greater for FFS than the other two foot strike patterns, and that the forces of MFS were greater than RFS. Furthermore, we found that the differences in these forces were mainly attributable to differences in the GRF moment arm among the three foot strike patterns. 


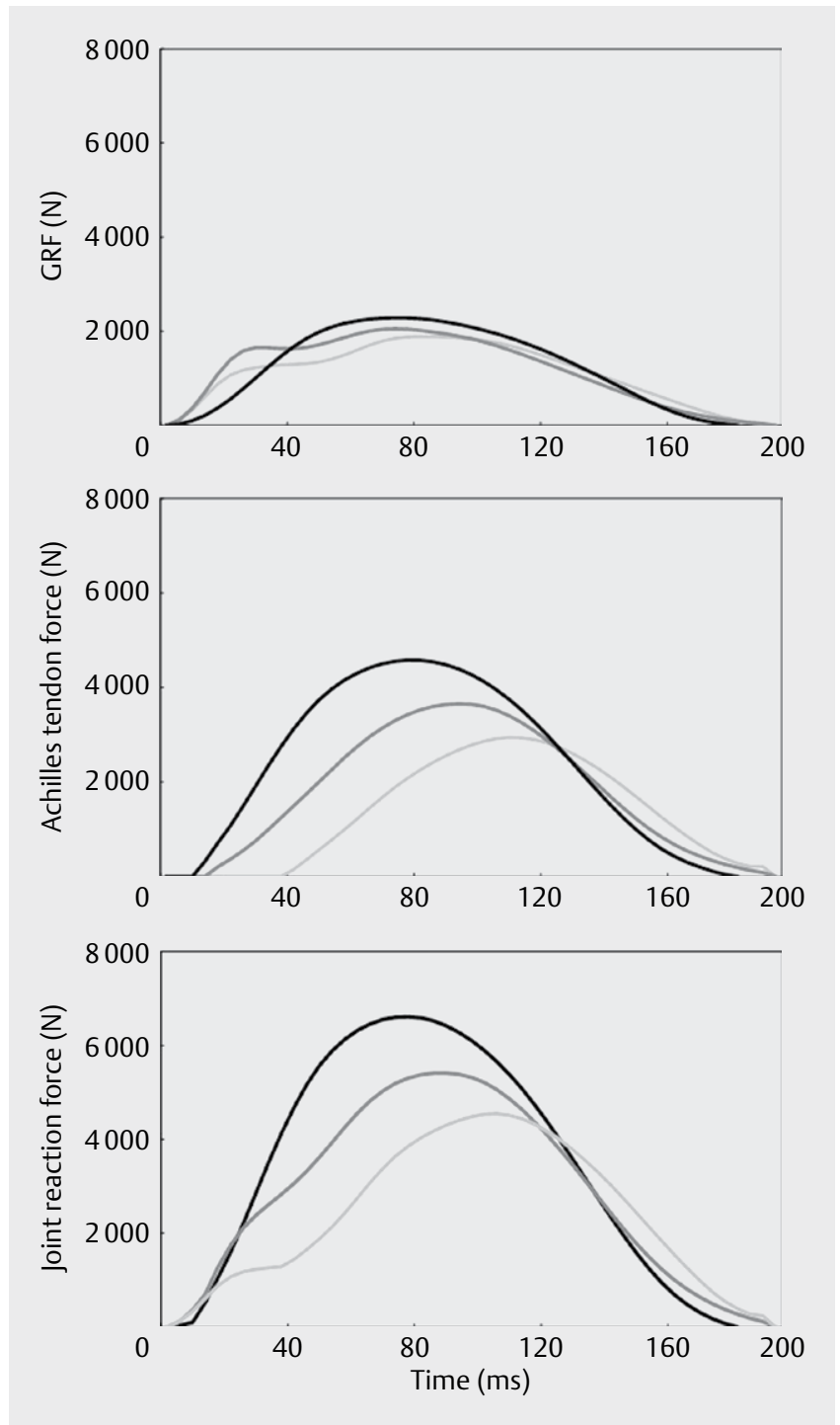

- Fig. 2 Typical GRF, Achilles tendon force and joint reaction force observed during the contact phase of running. Lines represent FFS (black), MFS (dark gray), and RFS (light gray) forces.

\section{Acknowledgements}

This study was partly supported by the Grant from the Descente and Ishimoto Memorial Foundation for the Promotion of Sports Science, and the Grant-in-Aid for Research Fellow of the Japan Society for the Promotion of Science (13J10708) and for Young Scientist B (15K16462).

\section{Conflict of Interest}

The authors declare that they have no conflict of interest.

\section{References}

[1] Ae M, Tan H, Yokoi T. Estimation of inertia properties of the body segments in Japanese athletes (in Japanese with English abstract). In: The Society of Biomechanisms. Biomechanisms 11. Tokyo: The University of Tokyo Press; 1992: 23-33

[2] Almonroeder T, Willson JD, Kernozek TW. The effect of foot strike pattern on Achilles tendon load during running. Ann Biomed Eng 2013; 41: 1758-1766

[3] Armstrong RB, Warren GL, Warren JA. Mechanicsms of exercise-induced muscle fiber injury. Sports Med 1991; 12: 184-207

[4] Braunstein B, Arampatzis A, Eysel P, Bruggemann GP. Footwear affects the gearing at the ankle and knee joints during running. J Biomech 2010; 43: 2120-2125

[5] Cavanagh PR, Lafortune MA. Ground reaction forces in distance running. J Biomech 1980; 13: 397-406

[6] Clarke EC, Martin JH, d'Entremont AG, Pandy MG, Wilson DR, Herbert RD. A non-invasive, 3D, dynamic MRI method for measuring muscle moment arms in vivo: demonstration in the human ankle joint and Achilles tendon. Med Eng Phys 2015; 37: 93-99

[7] Clausen JP. Circulatory adjustments to dynamic exercise and effect of physical-training in normal subjects and in patients with coronary-artery disease. Prog Cardiovasc Dis 1976; 18: 459-495

[8] Daoud AI, Geisseler G], Wang F, Saretsky J, Daoud YA, Lieberman DR. Foot strike and injury rates in endurance runners: A retrospective study. Med Sci Sports Exerc 2012; 44: 1325-1334

[9] Devita P, Fellin RE, Seay JF, Ip E, Stavro N, Messier SP. The relationships between age and running biomechanics. Med Sci Sports Exerc 2016; 48: 98-106

[10] Edwards WB, Gillette JC, Thomas JM, Derrick TR. Internal femoral forces and moments during running: implications for stress fracture development. Clin Biomech 2008; 23: 1269-1278

[11] Ferber R, Daveis IM, Williams DS $3^{\text {rd }}$. Gender differences in lower extremity mechanics during running. Clin Biomech 2003; 18: 350-357

[12] Fukunaga T, Roy RR, Shellock FG, Hodgson JA, Edgerton VR. Specific tendon of human plantar flexors and dorsiflexors. J Appl Physiol 1996; 80: $158-165$

[13] Gottschall JS, Kram R. Ground reaction forces during downhill and uphill running. J Biomech 2005; 38: 445-452

[14] Harriss DJ, Atkinson G. Ethical standards in sports and exercise science research: 2014 update. Int J Sports Med 2013; 34: 1025-1028

[15] Hasegawa H, Yamauchi T, Kraemer W]. Foot strike patterns of runners at the $15-\mathrm{km}$ point during an elite-level half marathon. J Strength Cond Res 2007; 21: 888-893

[16] Hashizume S, Fukutani A, Kusumoto K, Kurihara T, Yanagiya T. Comparison of the Achilles tendon moment arms determined using the tendon excursion and three-dimensional methods. Physiol Rep 2016; 4: e12967

[17] Hashizume S, Iwanuma S, Akagi R, Kanehisa H, Kawakami Y, Yanai T. In vivo determination of the Achilles tendon moment arm in three-dimensions. J Biomech 2012; 45: 409-413

[18] Hashizume S, Iwanuma S, Akagi R, Kanehisa H, Kawakami Y, Yanai T. The contraction-induced increase in Achilles tendon moment arm: a three-dimensional study. J Biomech 2014; 47: 3226-3231

[19] Jakobsen BW, Krøner K, Schmidt SA, Jensen J. Running injuries of exercise marathon: registration of claims records and types of damage at Aarhus Marathos. 1986; Ugeskr Laeg 1989; 151: 2189-2192

[20] Komi PV, Fukashiro S, Jarvinen M. Biomechanical loading of Achilles tendon during normal locomotion. Clin Sports Med 1992; 11: 521-531 
[21] Kulmala JP, Avela J, Pasanen K, Parkkari J. Forefoot strikers exhibit lower running-induced knee loading than rearfoot strikers. Med Sci Sports Exerc 2013; 45: 2306-2313

[22] Laughton CA, Davis I, Hamill J. Effect of strike pattern and orthotic intervention on tibial shock during running. J Appl Biomech 2003; 19: 153-168

[23] Lieberman DE, Venkadesan M, Werbel WA, Daoud AI, D’Andrea S, Davis IS, Mang'Eni RO, Pitsiladis Y. Foot strike patterns and collision forces in habitually barefoot versus shod runners. Nature 2010; 463: 531-535

[24] Lun V, Meeuwisse WH, Stergiou P, Stefanyshyn D. Relation between running injury and static lower limb alignment in recreational runners. Brit J Sport Med 2004; 38: 576-580

[25] Maganaris CN, Baltzopoulos V, Ball D, Sargent AJ. In vivo specific tension of human skeletal muscle. J Appl Physiol 2001; 90: 865-872

[26] Maughan RJ, Miller JD. Incidence of training-related injuries among marathon runners. Brit J Sport Med 1983; 17: 162-165

[27] Mitchell JH. Exercise training in the treatment of coronary heart disease. Adv Intern Med 1975; 20: 249-272

[28] Nilsson ], Thorstensson A.. Ground reaction forces at different speeds of human walking and running. Acta Physiol Scand 1989; 136: 217-227

[29] Perl DP, Daoud Al, Lieberman DE. Effects of footwear and strike type on running economy. Med Sci Sports Exerc 2012; 44: 1335-1343

[30] Queen RM, Abbey AN, Chuckpaiwong B. Nunley JA. Effects of footwear and strike type on running economy. Med Sci Sports Exerc 2012; 44: 1335-1343

[31] Rogers MA, Yamamoto C, Hagberg JM, Holloszy JO, Ehsani AA. The effect of 7 years of intense exercise training on patients with coronary-artery disease. J Am Coll Cardiol 1987; 10: 321-326

[32] Rooney BD, Derrick TR. Joint contact loading in forefoot and rearfoot strike patterns during running. J Biomech 2013; 46: 2201-2206
[33] Sasimontonkul S, Bay BK, Pavol M]. Bone contact forces on the distal tibia during the stance phase of running. J Biomech 2007; 40: 3503-3509

[34] Scott SH, Winter DA. Internal forces at chronic running injury sites. Med Sci Sport Exer 1990; 22: 357-369

[35] Sheehan FT. The 3D in vivo Achilles' tendon moment arm, quantified during active muscle control and compared across sexes. J Biomech 2012; 45: 225-230

[36] Shih Y, Lin KL, Shiang TY. Is the foot striking pattern more important than barefoot or shod conditions in running? Gait posture 2013; 38: 490-494

[37] Silvernail JF, Boyer K, Rohr E, Brüggemann GP, Hamill J. Running mechanics and variability with aging. Med Sci Sports Exerc 2015; 47: 2175-2180

[38] Taunton JE, Ryan MB, Clement DB, McKenzie DC, Lloyd-Smith DR, Zumbo BD. A prospective study of running injuries: the Vancouver Sun Run “In Training” clinics. Brit J Sport Med 2003; 37: 239-244

[39] van Gent RN, Siem D, van Middelkoop M, van Os AG, Bierma-Zeinstra SM, Koes BW. Incidence and determinants of lower extremity running injuries in long distance runners: a systematic review. $\mathrm{Br}$ J Sports Med 2007; 41: 469-480

[40] Vannatta CN, Kernozek TW. Patellofemoral joint stress during running with alternations in foot strike pattern. Med Sci Sports Exerc 2015; 47: 1001-1008

[41] Varnauskas E, Bergman H, Houk P, Björntorp P. Haemodynamic effects of physical training in coronary patients. Lancet 1966; 288: 8-12

[42] Wiegerinck JI, Boyd J, Yoder JC, Abbey AN, Nunley JA, Queen RM. Differences in plantar loading between training shoes and racing flats at a self-selected running speed. Gait Posture 2009; 29: 514-519

[43] Williams DS, McClay IS, Manal KT. Lower extremity mechanics in runners with a converted forefoot strike pattern. J Appl Biomech 2000; 16: $210-218$

[44] Yu B, Hay JG. Angular-momentum and performance in the triple jump: a cross sectional analysis. J Appl Biomech 1995; 11: 81-102 